\title{
Information Retrieval System Based on Context-aware in Internet of Things
}

\author{
Ma Junhong ${ }^{1, a^{*}}$ \\ ${ }^{1}$ Xi'an International University, Shaanxi, China, 710000 \\ asufeiya913@qq.com
}

Keywords: Context-aware computing, Internet of things, Information retrieval, Ontology

\begin{abstract}
The Context-aware computing in internet of things plays an important role in extracting useful information from a large amount of data. In this paper, through analysis on the characteristics of information in internet of things, using existing internet situation cognitive computing technology, the authors put forward an improved information retrieval method which could present the retrieval results through integration of situational factors into calculation, feature extraction and semantic representation. Finally, the experiments showed that the proposed system could improve the efficiency of information retrieval.
\end{abstract}

\section{Introduction}

At present, the Internet of things with big data, cloud computing, mobile Internet is regarded as the four main technologies, the Internet is the world's academic research in key areas. In 2015, as the global technology industry event - CES conference ended the arrival of the means of the first year of the Internet of things. Japan and South Korea respectively puts forward the "Japan" and "U Chesapeake" strategy, from the Angle of country, industry, science and technology development strategy of the important content of network information is put forward [1].

Attaches great importance to this too in our country, the national ministry of industry and information technology developed the "Internet of things" twelfth five-year "development plan; Build for IBM "smarter planet", the "experience China" was put forward in our country, and established the "experience China" centre in Wuxi; All the way "put forward by the chairman xi jinxing area" has become China's new international strategy framework, "One Belt And One Road" relying on infrastructure along each other, on the trade and the optimized configuration of production factors, so as to promote the development of regional integration. Each other to achieve infrastructure, tourism, agriculture, and trade and logistics development cannot leave the Internet of things in the field of cognitive information service technology.

As a new computing form, context aware computing is closely related to pervasive computing, mobile computing and intelligent computing. Generally speaking, research frontiers such as pervasive computing, collaborative computing, intelligent computing and virtual reality are all context aware[2]. This makes context aware computing a promising research area with vast applied prospects. With the developments of the Internet of things, sensor network, embedded system, communication network, distributed and mobile computing, context aware computing has drawn much attention from researchers both domestic and abroad. Through endowing the system the ability of sensing its surrounding environment, context aware computing optimizes the whole system (adjusting self behaviors, improving resource utilities), simplifies the interactions between the system and its users, improves users' experiences, makes the system intelligent, easy to use and user friendly.

\section{Information retrieval model}

The structure of information retrieval is an important part of information, analysis, organization, storage and retrieval. The purpose of information retrieval from a lot of information to find information to satisfy user needs [5]. Information retrieval processing object is the document 
resources, the content of the document including text, images, video, audio, and other forms. Figure 1 shows the information retrieval system structure, the concept of information retrieval includes three basic components: the query of the internal representation, internal documents, the match between the two, as shown in fig 1.

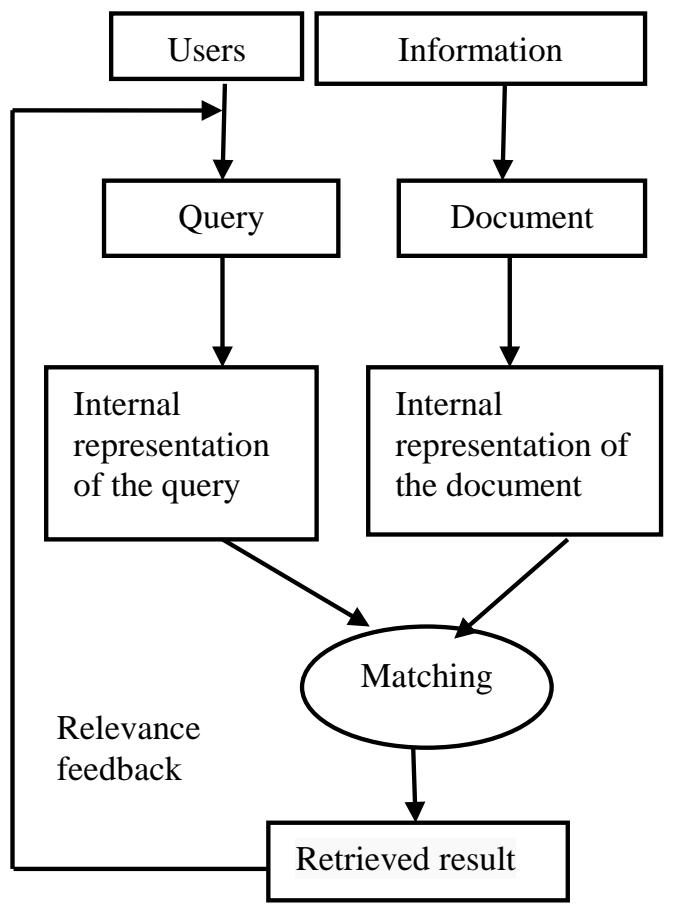

Fig 1. The Internet information retrieval system structure

\section{Context-aware information acquisition}

Context-aware information mainly obtained through sensors, Web Services, database and Web documents. Figure 2 shows situational information process.

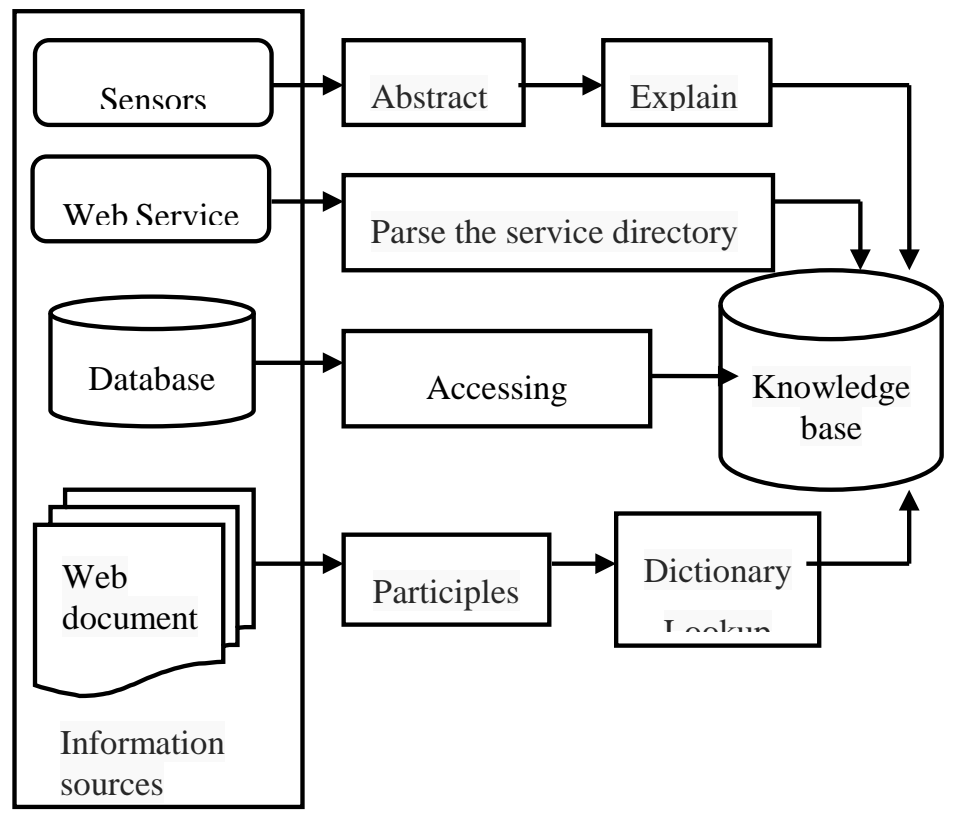

Fig 2. The workflow of acquiring contextual information

\section{A conceptual model of the scene management system}


Through comprehensive study, this paper design the scene management system model is divided into three layers. The underlying layer perceptions and situational resources, means different sensors and situational resources. Intermediate level for situation management system, it includes query, storage and pre-treatment layer. For the application layer at the top, said a variety of applications, such as location services, semantic query. In the middle of the management system, first of all, through the preliminary situational context modelling to determine the application data model, if there is a application interaction and data sharing, requires a unified model of the situation. In the middle of the management system, that receive or active in the extraction of the lower perception and situation of resources to provide situation data. Through pre-processing module, data management system first filtered and converted into a unified format and units, and then according to the situation the semantic matching model and hierarchical classification and clustering, if it is multiple applications share situation requires further consolidation. Clustering or consolidated situation after data is submitted to the storage module. These contextual information storage modules are responsible for efficient storage. Upper multiple applications can be accessed by subscription and query distribution and query module. Distribution and query module content analysis to subscribe to and query situation, content to read according to the situation and distribution or return possible situation data.

Basic tree hierarchy for object attributes are classified according to a single type of situation cannot effectively reflect the properties of the type according to multi-dimensional situation of object clustering and classification process. In this paper, we used multiple attribute tree combined with concept lattice to query, update, segmentation, etc.

\section{Layered architecture of the System}

Layered architecture of the System is as figure 3:

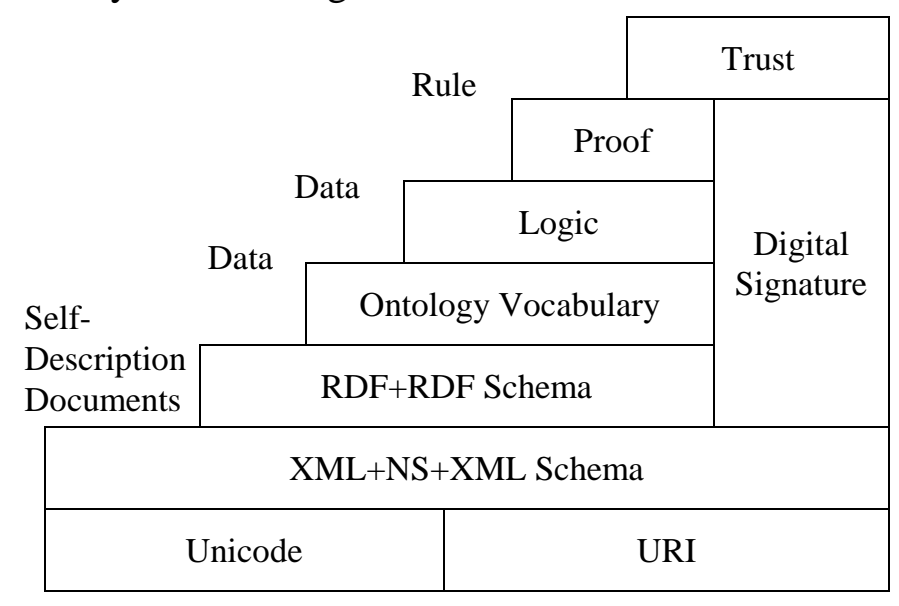

Fig3. Layered architecture

1. Unicode and URI: In the architecture of the semantic web, Unicode is mainly responsible for coding for processing of resources, URI is mainly responsible for the resources for a unique identifier, and the layer is the foundation of the semantic web architecture

2.XML+NS +XML Schema: This layer is mainly through the use of standard language separation Web information content of data structure and expression, can come up from the syntax structure and content of representing data.

3. RDF+RDF Schema: RDF (Resource Description Framework) is a basic Resource Description model, it contains a triple (objects, attributes and attribute values) statement, it is the most basic Web semantic expression of specification. RDF Schema (RDFS) through provides the basis for the RDF data model of the term, as the object of Web resources and build hierarchical structure.

4. Ontology Vocabulary: This layer is defined on the basis of RDF and RDFS layer of ontology concepts. The layer of ontology is abstract description of concepts and relationships between rich 
concepts, is mainly used to express the knowledge in the field of computer application, which can be extended vocabulary.

5. Logic、Proof and Trust: Logic layer is mainly provide some axioms and inference rules of ontology, the ontology in semantic Logic and knowledge. Knowledge expression can expand and promote, but also can express dynamic knowledge in the field of application. Proof layer consists of the validation method and process of reasoning, said the main is the application of knowledge and rule reasoning. After establishing Logic, can the concept, the relationship between concepts and reasoning results validated by logical reasoning, to demonstrate the effectiveness of the reasoning. Trust layer is used to implement the Trust management mechanism in the network environment, it can use the certificate or digital signature, based on intelligent agent can also be used between community members and recommend ways to achieve.

\section{Experimental analysis}

In this paper, the traditional method based on keywords retrieval, statistical method and information extraction method based on the situation of application, data from a logistics canter of user query data. Application interaction related objects, also including the user and the application itself. "Because the scene is a very broad concept, so a suitable classification system will contribute to understanding of the situation, the management and use. At present, for there are many types of the classification of the scene, according to the characteristics of the Internet of data, this is divided into five categories:

1.Computing the Context: available equipment, network connectivity and communication overhead; 2. The User Context: User basic information, interest preference and the relationship between users;

3. The Physical Context: light, temperature and sound;

4. The Time Context: when, day, month, and year;

5. Social Context, habits, customs, rules and laws.

After many experiments, results in the following table 1:

Tab 1. The experiments results

\begin{tabular}{|c|c|c|c|}
\hline & P@10 & P@15 & P@20 \\
\hline $\begin{array}{c}\text { statistical } \\
\text { methods }\end{array}$ & 0.463 & 0.622 & 0.549 \\
\hline keyword search & 0.582 & 0.687 & 0.713 \\
\hline $\begin{array}{c}\text { Context-aware } \\
\text { computing }\end{array}$ & 0.765 & 0.842 & 0.865 \\
\hline
\end{tabular}

This article USES the P@ N to measure the efficiency of the algorithm.P@ N is short for Precision @ $\mathrm{N}$; P is for a particular query, location factors, considering the search results before testing article $\mathrm{N}$ the accuracy of the results [6]. When demand is gradually increasing n, P @ n will approached precision. The P@ N computation formula is as follows:

$$
\mathrm{P} @ \mathrm{~N}=\frac{\sum_{\mathrm{i}=1}^{\mathrm{M}}(\mathrm{Pi})}{\mathrm{M}}
$$

In order to give full consideration to situational factors, we joined the feature information query. Compared with the recall rate, the precision rate is more important for the users who want to obtain the information of context-aware and would be satisfied with its higher precision rate.

\section{Conclusions}

It is can be seen from the above experimental data, this paper studies the method compared with the traditional method has a higher efficiency of information extraction. Along with the Internet of things, 
semantic computing, the further development of intelligent technology such as dressing, situational awareness technology faces more challenges. Is widely used in this paper, the research results, not only can be used for the Internet of things intelligent retrieval, but also to the intelligent logistics, intelligent transportation, intelligent learning system, and the mobile terminal, based on the location of the perception of information retrieval, WEB application, service-oriented semantic mining area also has a certain reference value.

At present, with the development of context-aware computing, people for privacy protection, especially the user's location privacy protection is more and more attention. However users' privacy protection is not alone can solve the problem of a module; each section will be involved in the system. This requires in context-aware computing framework with a content of the user privacy protection. This is also this topic later research direction and important content.

\section{Acknowledgment}

This work is financially supported by the natural science foundation research project fund of Shaanxi provincial science and technology department of China (Grant NO. 2014JM8323). And the Xi'an Social Science Foundation : Study on the way from Xi'an university network public opinion guide in media era.

\section{References}

[1] Junzhong Gu, Gong-Chao Chen. Design of Physical and Logical Context Aware Middleware [J].International Journal of Signal Processing, Image Processing and Pattern Recognition,2012,(1):113一130.

[2] Lin Y, Zhang J., Chung, H. S. etc., An Ant Golony Optimization Approach for Maximizing Irifetime of Heterogeneous Sensor Networks. IEEE Trans. Systems, Man, and Cybernetics-PART C, 42(3), 2012, p.408-420.

[3] Dhillon S. S., Vanmieghem P., Performance analysis of the AntNet Algorithm,51(8), 2007; p.2104-2125.

[4] Loyall J P, Schantz R E. Using Context Awareness to Improve Quality of Information Retrieval in Pervasive Computing[C].IFIP International Federation for Information Processing 009:320-331.

[5] Yue Suo, Naoki Miyata, Toru Ishida, Yuanchun Shi. Open Smart Classroom: Extensible and Scalable Learning System in Smart Space Using Web Service Technology [J]. IEEE Transactions on Knowledge and Data Engineering, 2009, (6): 814-828.

[6] SUN HaoJun, SHAN GuangHui, GAO YuLong. "Algorithm for high-dimensional categorical data weighted subspace clustering”, Computer Engineering and Applications, vol. 50, Issue 23, 2014, pp. 131-135. 